\title{
Dynamical tunneling in molecules: role of the classical resonances and chaos
}

\author{
Srihari Keshavamurthy \\ Department of Chemistry, Indian Institute of Technology, Kanpur, India 208016
}

(Dated: November 13, 2018)

\begin{abstract}
In this letter we study dynamical tunneling in highly excited symmetric molecules. The role of classical phase space structures like resonances and chaos on the tunneling splittings are illustrated using the water molecule as an example. It is argued that the enhancements in the splittings due to resonances (near-integrable phase space) and due to chaos (mixed phase space) are best understood away from the fluctuations associated with avoided crossings. In particular we provide an essential difference between the two mechanisms in terms of high order perturbation theory. The analysis, apart from testing the validity of a perturbative approach, suggests such systems as prime candidates for studying dynamical tunneling.
\end{abstract}

The concept of dynamical tunneling emerged more than two decades ago in the context of studying near degenerate vibrational states of symmetric molecules. As an example in the water molecule the symmetry of the $\mathrm{OH}$ bonds implies that a state $\left(n_{1}=3, n_{2}=0\right)$ which has three quanta of excitation in one of the $\mathrm{OH}$ bond $\left(n_{1}=3\right)$ and zero quanta of excitation in the other equivalent $\mathrm{OH}$ bond $\left(n_{2}=0\right)$ is degenerate with the state $(0,3)$. The two states are not coupled through any classical process i.e., a classical trajectory started with initial conditions corresponding to the state $(3,0)$ will remain localized indefinitely without ever evolving to the part of the phase space corresponding to the state $(0,3)$. However, Lawton and Child[1] realized that a generalized form of tunneling mixes the two degenerate states giving rise to a characteristic splitting. In an influential work Davis and Heller [2] refined and generalized the earlier observations and introduced the term dynamical tunneling to distinguish it from the usual tunneling through potential barriers in coordinate space. One of the important outcomes of the study was the suggestion that the underlying phase space of the system is the proper setting to understand dynamical tunneling.

In an apparently unrelated developement researchers studying the problem of intramolecular energy redistribution (IVR) discovered purely quantum energy flow between modes which would be otherwise uncoupled. A detailed analysis by Hutchinson, Sibert and Hynes $3[$ established that the mechanism for such clasically forbidden energy flow between degenerate vibrational modes arose from indirect state-to-state explorations involving a sequence of intermediate states. Important insights were provided by Stuchebrukhov and Marcus 泪 who established that one could view dynamical tunneling as a high order perturbative process involving a sequence of offresonance virtual states ("vibrational superexchange"). Interestingly it was also shown that the perturbative approach to the tunneling splitting was related in a simple way to the usual semiclassical JWKB solution.

Despite this important prior work the explicit demonstration of the role of various classical phase space structures in dynamical tunneling has only recently been es- tablished. In particular the work of Bohigas, Tomsovic and Ullmo elucidating the role of chaos on dynamical tunneling has led to a resurgence of interest in the field. A key observation that emerged from numerous studies [6] of chaos-assisted tunneling (CAT) is that such a process necessarily requires atleast a three level mechanism. One of the hallmarks of CAT has to do with erratic fluctuations of the tunnel splittings with variations in energy or system parameters. The fluctuations were explained on the basis of a tunnel doublet, associated with regular regions in the phase space, invoved in an avoided crossing with a third state inhabiting the chaotic region of the phase space.

However, very recently classical nonlinear resonances in the near-integrable phase space regimes have also been implicated to play a dominant role in dynamical tunneling [7, 8]. This has been dubbed, in analogy with CAT, as resonance-assisted tunneling (RAT) and studies revealed that tunnel splitting fluctuations, possibily more intense than in CAT, occur in near-integrable systems as well due to crossing of the regular tunnel doublets with a regular third state. The sheer richness of the phase space perspective in dynamical tunneling is further exemplified by the work of Frischat and Doron[9]. At present the consensus regarding dynamical tunneling seems to be that CAT and RAT are manifestations of the more general phenomenon of transport-assisted tunneling[9].

The preceeding discussion makes it clear that splitting fluctuations can occur in general nonintegrable systems and tunneling can be ascribed to chaos or resonances depending on the phase space nature of the third 'intruder' state. It therefore seems natural to focus attention away from the avoided crossings in order to gain insights into the process 88]. The present work is concerned with the study of dynamical tunneling in molecules from such a perspective. An essential difference from the work of Brodier, Schlagheck and Ullmo 8 is that the focus in this work is entirely on a perturbative evaluation of the splittings to quantitative accuracies. The splittings $\Delta$ associated with the doublet states $|i\rangle$ and symmetry related $|f\rangle$ are calculated by high order nondegenerate perturba- 


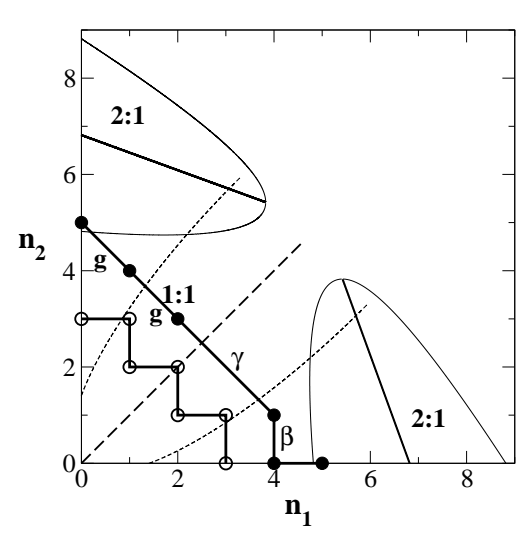

FIG. 1: State space and the location of the various resonances for water with $P=8$. The $2: 2$ zone has been supressed for clarity. Two minimal perturbative paths $\Gamma_{600}$ (open circles) and $\Gamma_{221}$ (filled circles) in state space are shown as examples.

tion theory involving the unperturbed states $\left\{\alpha_{r}\right\}$ as

$$
\frac{\Delta}{2}=\sum_{r \neq i, f} \frac{V_{i 1}^{(1)} V_{12}^{(2)} \ldots V_{n f}^{(n)}}{\Delta E_{i 1} \Delta E_{i 2} \ldots \Delta E_{n f}},
$$

where $\widehat{V}^{(r)}$ are the local perturbations that connect the intermediate states through $V_{q r}^{(r)}=\left\langle\alpha_{q}\left|\widehat{V}^{(r)}\right| \alpha_{r}\right\rangle$ with $\Delta E_{i r}=\left(E_{i}-E_{r}\right)$. The sum over the intermediate states can be viewed as a sum over paths in the state space and the number of intermediate states is related to the length of a path. The perturbative treatment is valid as long as $V_{q r}^{(r)} / 2<<\Delta E_{i r}$ and hence the method cannot be used to calculate splittings for states involved in avoided crossings. In principle, an infinite number of high order perturbative chains (paths in state space) exist which connect the two degenerate states. The hope has been that perhaps a few such paths would dominate the dynamical tunneling in the integrable and near-integrable cases. In this work we show that even in near-integrable cases there is no single dominant perturbative path. However, it is possible to identify a certain family of perturbative chains ("minimal paths" in state space) that are sufficient to provide quantitative results. Parameteric variations tune the phase space from the near-integrable limit to the mixed regime and it is observed that the minimal paths are not sufficient anymore to reproduce the splittings.

The purpose of this letter is to illustrate the observations above and accentuate the various mechanisms of dynamical tunneling. The system of choice is the water molecule which can be described by an effective spectroscopic Hamiltonian 10]

$$
\widehat{H}_{\text {eff }}=\widehat{H}_{0}+g \widehat{V}_{1: 1}^{(12)}+\gamma \widehat{V}_{2: 2}^{(12)}+\beta\left(\widehat{V}_{2: 1}^{(1 b)}+\widehat{V}_{2: 1}^{(2 b)}\right),
$$

where $\widehat{H}_{0}$ is diagonal in the number basis $\left(n_{1}, n_{2}, n_{b}\right)$ and $g, \gamma, \beta$ represent the strengths of the various perturbations. The various parameters of $\widehat{H}_{\text {eff }}$ are given in the work of Baggott 10]. The zeroth order quantum numbers $\left(n_{1}, n_{2}, n_{b}\right)$ represent the excitation quanta in the two, equivalent $\mathrm{OH}$-stretches and the bend mode respectively. The perturbations $\widehat{V}$ are off-diagonal in the number basis and have the form:

$$
\widehat{V}_{m: n}^{(i j)}=\left[\left(\hat{a}_{i}\right)^{n}\left(\hat{a}_{j}^{\dagger}\right)^{m}+\text { h.c. }\right]
$$

where $\hat{a}_{i}$ and $\hat{a}_{i}^{\dagger}$ are the annhilation and creation operators for the mode $i$. The resonant perturbations $\widehat{V}_{m: n}^{(i j)}$ are responsible for the exchange of quanta (energy) between the modes $i$ and $j$. Classical-quantum correspondence studies 11] have established that the classical limit Hamiltonian corresponding to $\widehat{H}_{\text {eff }}$ is nonlinear and multiresonant. The location of the various resonance zones 11] in state space are shown in Fig. (17). Note that

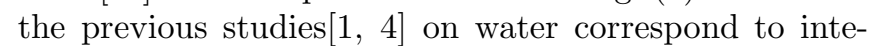
grable phase space regimes.

Clearly $\widehat{H}_{\text {eff }}$ is symmetric under $n_{1} \leftrightarrow n_{2}$ and $P \equiv$ $n_{1}+n_{2}+n_{b} / 2$ is a conserved quantity. Consequently $\widehat{H}_{\text {eff }}$ for a given $P$ is of the order $(P+1)(P+2) / 2$ making the numerical investigations rather straightforward. In addition $\widehat{H}_{\text {eff }}$ exhibits a wide range of behaviour from integrable to near-integrable to mixed classical dynamics. Due to the exact $C_{2 v}$ symmetry tunneling doublets exist over the entire range of the classical dynamics. In this work six tunneling doublets with $P=8$, denoted by $\left|m, \pm m, n_{b}\right\rangle$ with $m=n_{1}+n_{2}$ are studied. The labels used for the states are appropriate in the absence of the $2: 1$ resonances. We will continue to use them for convenience keeping in mind that the actual assignments of these states are different 11. The energies of the states approximately span the range $[24256,25574] \mathrm{cm}^{-1}$ above the ground state. For comparison the various resonant strengths have the typical values $\beta \approx 27 \mathrm{~cm}^{-1}, g \approx-50$ $\mathrm{cm}^{-1}$ and $\gamma \approx-1.0 \mathrm{~cm}^{-1}$.

Reperesentative phase space sections are shown in Fig. (2) for the highest energy state among the doublets considered. In Fig. (3) the doublet splittings, $\Delta_{m}$, are shown for the various states $\left|m, \pm m, n_{b}\right\rangle$. Note that the states in Fig. (3) are not energy ordered and in particular the state $|8, \pm 8,0\rangle$ is lower in energy than the state $|5, \pm 5,6\rangle$. The splittings for the integrable cases (only $1: 1$ and 1:1+2:2) are shown in the inset to Fig. (3). As expected [4] the splittings show a monotonic decrease with increasing amount of stretch excitation and no fluctuations are observed. Even within the integrable cases it is relevant to note that the addition of the weak $2: 2$ results in a significant increase in $\Delta$. High order perturbation calculation 四 essentially reproduces the splittings to quantitative accuracy as is apparent from the inset in Fig. (3). Inclusion of the 2:1 stretch-bend resonances renders the system nonintegrable and clear deviations from the integrable cases are seen. In the main part of Fig. (3) the splittings are shown as one proceeds from near-integrable to the full, mixed scenario. The nature of 


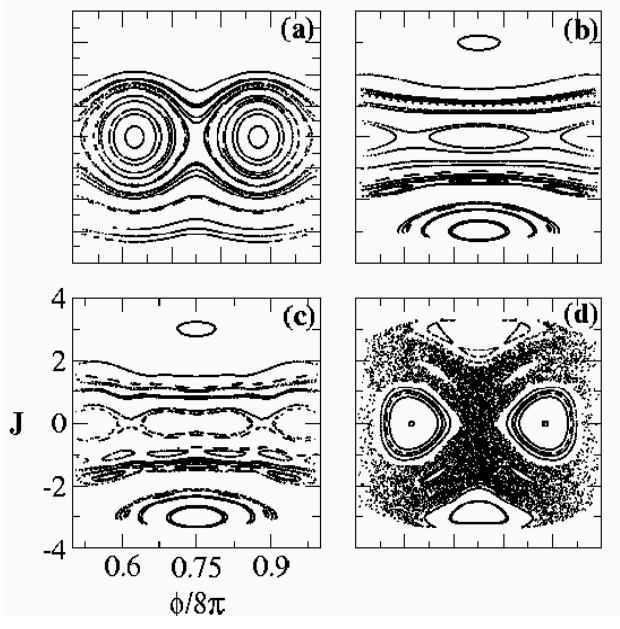

FIG. 2: Poincaré surface of sections at energy corresponding to the doublet $|6, \pm 6,4\rangle$. The action variable $J=\left(I_{1}-I_{2}\right) / 2$ and the conjugate angle variable $\phi=\theta_{1}-\theta_{2}$. (a) Integrable 1:1+2:2 (b) Near-integrable 2:1 (c) Near-integrable 2:1+2:2 and (d) full system. The 2:1 islands are centered at $\phi=6 \pi$ and $J \approx \pm 3$ while the $1: 1$ islands appear around $J=0$. The surface of section is symmetric with respect to reflection about the $J=0$ axis.

the underlying phase space clearly reflects the transition from near-integrable to the mixed regime (cf. Fig. (2bd)). The main difference from the integrable case is the nonmonotonic behaviour of $\Delta_{m}$. The intense fluctuations for the state $|7, \pm 7,2\rangle$ are associated with its proximity to an avoided crossing. The key observation here is that the fluctuation due to avoided crossing is a robust feature and persists in the full system. Indeed it has been confirmed that in the $2: 1$ only case there is a strong doublet-doublet crossing which transforms into a singlet-doublet crossing in the full system and for the deuterated analog $\mathrm{D}_{2} \mathrm{O}$ a similar calculation shows absence of fluctuations in the full system despite the presence of significant chaos 12 . Thus it is crucial to shift the focus away from the fluctuations to uncover the effect of chaos and resonances on dynamical tunneling in the system.

In Fig. (3) the phenomenon of RAT is evident in going from the 2:1 only system to the $2: 1+2: 2$ system. The enhancements of the splittings are even more dramatic considering the fact that states with odd $m$ do not split at all if only the 2:2 resonance is present. Similarly the enhancement of the splittings on going from the $2: 1+2: 2$ to the full system can be ascribed to CAT. Note that the enhancement due to CAT are typically an order of magnitude smaller than those due to RAT as has been earlier observed in the annular billiard studies [9]. A crucial difference between CAT and RAT arises from the perturbative viewpoint. In order to highlight this difference we begin by noting that the structure of the resonances imply, in terms of order, $\beta^{4} \sim g^{2} \sim \gamma$. Hence a consistent perturbative calculation of the splitting for the state

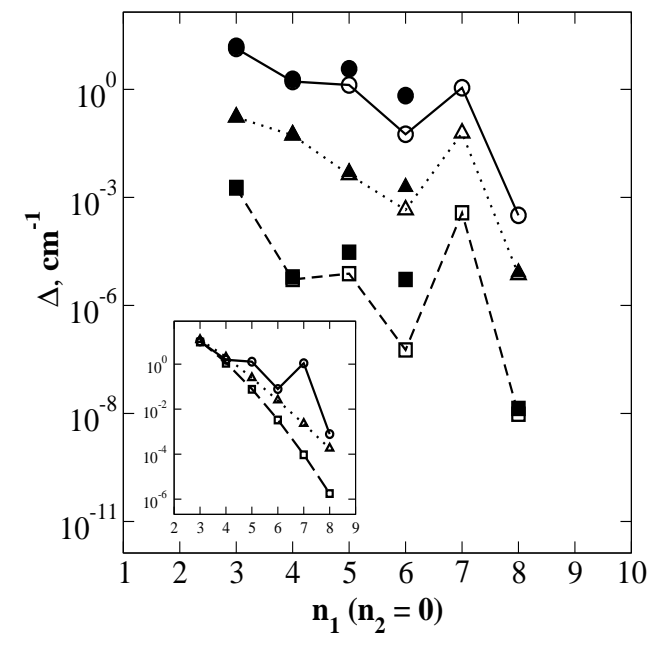

FIG. 3: Dynamical tunneling splittings for the nonintegrable cases. Squares (only 2:1), triangles $(2: 1+2: 2)$, and the full system (circles). The perturbative results (converged) using minimal paths are shown by filled symbols. Mean level spacings $\approx 100 \mathrm{~cm}^{-1}$. (Inset) Splittings for the integrable cases of the $1: 1$ (squares) and 1:1+2:2 (triangles). The high order perturbation results are shown as dashed and dotted lines respectively. The full case is also shown for comparison.

$\left|m, \pm m, n_{b}\right\rangle$ is obtained as:

$$
\Delta_{m}=\sum_{a, b, c} \beta^{a} g^{b} \gamma^{c} \sum_{n} \Delta_{m}\left(\Gamma_{a b c}^{(n)}\right)
$$

where $n$ indexes all possible paths $\Gamma_{a b c}$ for a particular choice of $a, b, c$ satisfying the constraint $a+2 b+4 c=2 m$. It is clear that all possible paths have not been included in the above calculation of $\Delta_{m}$. The paths included, called minimal paths, are all of the effective order $2 m$. Examples of the minimal paths $\Gamma_{600}$ and $\Gamma_{221}$ relevant for calculating $\Delta_{3}$ and $\Delta_{5}$ respectively are shown in Fig. (1). One of the observations from this work is that for nearintegrable systems a particular family of the minimal paths is dominant. In contrast, more than one family and higher order paths are required in the presence of significant chaos. As an example consider the case of $\Delta_{5}$. This state is involved in a weak avoided crossing and hence provides a stringent test for the nondegenerate perturbation theory. In the near-integrable cases the family of paths $\Gamma_{1000}, \Gamma_{202}$, and $\Gamma_{601}$ are sufficient. Perturbative calculations yield the splittings $\Delta_{5}\left(\Gamma_{1000}\right)=6.1 \times 10^{-5}$ $\mathrm{cm}^{-1}$ and $\Delta_{5}\left(\Gamma_{202,601}\right)=7.2 \times 10^{-3} \mathrm{~cm}^{-1}$ as compared to the numerically exact values of $1.7 \times 10^{-5}$ and $6.2 \times 10^{-3} \mathrm{~cm}^{-1}$ respectively. As mentioned earlier the $2: 1$ only system is less accurate due to the avoided crossing. In the integrable cases the perturbative results $\Delta_{5}\left(\Gamma_{050}\right) \approx 7.0 \times 10^{-2}$ and $\Delta_{5}\left(\Gamma_{050,031,012}\right) \approx 0.23 \mathrm{~cm}^{-1}$ are in good agreement with the exact values of $6.7 \times 10^{-2}$ and $0.23 \mathrm{~cm}^{-1}$ respectively. However, for the full system neither the near-integrable nor the integrable families are sufficient to reproduce the splitting $\left(\approx 1.6 \mathrm{~cm}^{-1}\right)$. In fact 
the families $\Gamma_{430}, \Gamma_{240}, \Gamma_{221}$ play an important role and yield a splitting of about $3.4 \mathrm{~cm}^{-1}$. For mixed systems, in general, a large number of families in the minimal set as well as higher order paths have to be included for a quantitatively accurate calculation of $\Delta_{m}$. Thus, for instance, a minimal path calculation of $\Delta_{6}$ yields a splitting which is about an order of magnitude large. The correlation of the preceeding observation with increasing amount of chaos in the phase space is an interesting possibility 12. As an example we mention that tuning the parameter $\gamma$ from about $1 \rightarrow 4 \mathrm{~cm}^{-1}$ turns the nearintegrable $2: 1+2$ : 2 system into a mixed system. The splitting $\Delta_{5}$ is seen to increase by about a factor of 22 whereas the minimal perturbative calculation predicts an accelaration by a factor of 12 . Clearly one anticipates higher order paths to play a significant role in mixed systems.

In conclusion we have shown that molecular systems with symmetry are prime candidates to study both chaos and resonance assisted dynamical tunneling [13]. High resolution spectroscopic studies of rovibrationally excited states are typically analysed in terms of such effective Hamiltonians [14]. The splitting patterns are crucial for understanding the extent of IVR in the molecule and hence the importance of dynamical tunneling[15]. This work is the first one to perform a detailed analysis of the possible mechanisms of dynamical tunneling in molecules with the hope that both CAT and RAT can be experimentally observed in the high resolution spectra. We have argued that a clear distinction between CAT and RAT can be established by focusing attention away from the avoided crossings. In addition high order perturbation theory, when valid, is quite successful in reproducing the tunnel splittings in the case of integrable and near-integrable systems. A single family of perturbative paths is sufficient but it is important to point out that among the many individual paths possible there is no single dominant path. For instance in the calculation of $\Delta_{5}$ the family $\Gamma_{1000}$ has 242 paths which come with both positive and negative contributions and one needs to sum over all the paths to obtain accurate splittings. The indications are that more than one family of paths including those of order larger than the minimal order is needed as soon as there is a significant amount of chaos in the system. The situation is reminiscent of the multistep indirect paths invoked in the annular billiard studies[9]. This provides important clues to the underlying mechanism of dynamical tunneling in the system. However, a semiclassical viewpoint on these perturbative minimal paths would lead to better insights into the process of dynamical tunneling from a phase space viewpoint.

It is a great pleasure to acknowledge Arul Lakshminarayan for helpful and critical discussions. This work was supported by funds from the Department of Science and Technology and the Council for Scientific and Industrial Research, India.

[1] R. T. Lawton and M. S. Child, Mol. Phys. 37, 1799 (1979).

[2] M. J. Davis and E. J. Heller, J. Chem. Phys. 75, 246 (1981).

[3] J. S. Hutchinson, E. L. Sibert III, and J. T. Hynes, J. Chem. Phys. 81, 1314 (1984).

[4] A. A. Stuchebrukhov and R. A. Marcus, J. Chem. Phys.98, 8443 (1993).

[5] O. Bohigas, S. Tomsovic, and D. Ullmo, Phys. Rep. 223, 43 (1993).

[6] Tunneling in Complex Systems, edited by S. Tomsovic (World Scientific, Singapore, 1998) and references therein; W. A. Lin and L. E. Ballentine, Phys. Rev. Lett. 65, 2927 (1990); R. Utermann, T. Dittrich, and P. Hänggi, Phys. Rev. E 49, 273 (1994).

[7] R. Roncaglia, L. Bonci, F. M. Izrailev, B. J. West, and P. Grigolini, Phys. Rev. Lett. 73, 802 (1994); L. Bonci, A. Farusi, P. Grigolini, and R. Roncaglia, Phys. Rev. E 58, 5689 (1998).

[8] O. Brodier, P. Schlagheck, and D. Ullmo, Phys. Rev. Lett. 87, 064101 (2001); O. Brodier, P. Schlagheck, and D. Ullmo, arXiv:nlin.CD/0205054.

[9] E. Doron and S. D. Frischat, Phys. Rev. Lett. 75, 3661 (1995); S. D. Frischat and E. Doron, Phys. Rev. E 57, 1421 (1998).

[10] J. E. Baggott, Mol. Phys. 65, 739 (1988).

[11] S. Keshavamurthy and G. S. Ezra, J. Chem. Phys. 107, 156 (1997).

[12] S. Keshavamurthy, to be published.

[13] Chaos-assisted tunneling has also been implicated in a molecule with internal rotation. See J. Ortigoso, Phys. Rev. A 54, R2521 (1996).

[14] M. Gruebele, Adv. Chem. Phys. 114, 193 (2000); G. S. Ezra, Adv. Class. Traj. Meth. 3, 35 (1998).

[15] E. J. Heller, J. Phys. Chem. 99, 2625 (1995). 